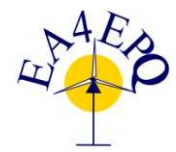

International Conference on Renewable Energies and Power Quality (ICREPQ'13)

Bilbao (Spain), 20 ${ }^{\text {th }}$ to $22^{\text {th }}$ March, 2013

Renewable Energy and Paver Quality. Fournal (RE\&PQJ)

ISSN 2172-038 X, No.11, March 2013

\title{
Design and Implementation of a High Temperature Control Monitoring Applied to Micro Thermoelectric Generators
}

\author{
S. C. S. Jucá ${ }^{1}$, P.C.M. Carvalho ${ }^{2}$, R. I. S. Pereira ${ }^{2}$, D. Petrov ${ }^{3}$ and U. Hilleringmann ${ }^{3}$ \\ ${ }^{1}$ Federal Institute of Education, Science and Technology of Ceará, IFCE - Contorno Norte Avenue, 10 \\ CEP: 61925-315, Maracanaú , CE (Brazil) \\ Phone/Fax number: +558533786329/+558533786311, e-mail: sandrojuca@ifce.edu.br \\ ${ }^{2}$ Department of Electrical Engineering \\ PICI CAMPUS, Federal University of Ceará - UFC DEE (Brazil) \\ e-mail: carvalho@dee.ufc.br, r.imaculada27@gmail.com \\ ${ }^{3}$ Universität Paderborn - UPB, Fachgebiet Sensorik, 33100, Paderborn, Germany \\ e-mail: petrov@sensorik.upb.de, hilleringmann@ieee.org
}

\begin{abstract}
The present paper describes the design and the implementation of a high temperature control monitoring (HTCM) for investigating the characteristics of a new type of thermoelectric generator (TEG) materials. This new material should work with temperature difference up to $400 \mathrm{~K}$, while most common TEGs using tellurium material operate with temperature differences up to $200 \mathrm{~K}$, and cannot withstand temperatures higher than $473 \mathrm{~K}$. In order to perform exact temperature control, the thermal power supplied to the HTCM and to regulate the temperature difference on TEG, a mathematical model of digitalized linear AC voltage phase switching was worked out and implemented in the developed device. This model makes it possible to achieve the exact accuracy of temperature control through the digital control of AC voltage phase switching. During the TEG performance measurement process the developed HTCM keeps the TEG hot side at the specified temperature, while the cold side is holding on constant temperature of approximately $293 \mathrm{~K}$. The HTCM embedded system was developed in order to test new TEG materials by specified high temperature ranges and to acquire and transmit data to an online host PC.
\end{abstract}

\section{Key words}

Online Monitoring, Thermoelectric Power Generation, Embedded System.

\section{Introduction}

The rising number of problems with electric energy generation from fossil fuels such as the carbon dioxide accumulation, air pollution and global warming together with their limited potential put forward the vital importance of the development of alternative energy technologies. As an environment-friendly energy source, thermoelectric power generation has attracted considerable interest due to its small size, zero carbon emission, absence of moving parts, low acoustic noise, high reliability and environmental energy production.
Thermoelectric power generators are also a good choice to produce electric power by recycling waste thermal energy [1].

Thermoelectric systems can be designed either for using a big amount of energy (geothermal energy [2] for example) or for working with small heat sources, used in automotive or home applications for waste heat recovery. Thermoelectric generators have even been miniaturized to absorb body heat for powering small electronic devices like a wristwatch [3].

The thermoelectric power generation is based on the Seebeck effect, who observed electric current flow on a junction of two different metals, joined at two points. One of them was heated while the other one was kept at a lower temperature. Thermoelectric energy production is one of the main processes of converting thermal energy directly into electrical energy, and ensure a long reliable operation without maintenance due to the nonexistence of moving parts [4]-[9]. Thermoelectric modules can be formed by $\mathrm{P}$ and $\mathrm{N}$ type semiconductors which are connected in series electrically and parallel thermally among two ceramic layers. A TEG is made by heating one side and cooling the other side of the thermoelectric module and connecting a load to the module end points. A basic schematic of a TEG is shown in Figure 1.

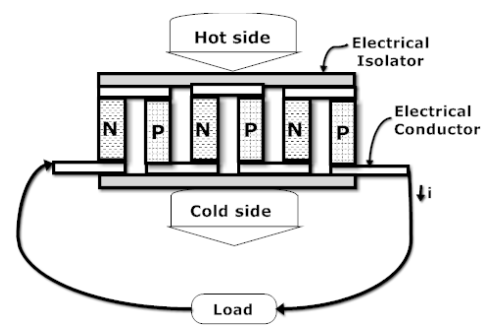

Fig. 1. Basic schematic of a thermoelectric generation process. 
Electrons on the material hot side are more energized than on the cold side. These electrons will flow from the hot to the cold side by connecting the both sides with a conductor wire. These electrons produce the electrical current. Connecting many thermocouples in series is possible to increase the output voltage and the output power [1]. The micro thermoelectric generators are used also by North American Space Agency (NASA) to supply electric energy to the Curiosity robot on Mars, through the heat liberated in radioisotope nuclear decay reactions [10], and as a source of renewable electric cogeneration through the heat supplied by incinerated municipal solid waste, automobile engines or industrial machinery [11].

This paper describes the temperature control development and data transmission equipment for evaluating of new TEG materials operating with higher temperatures difference than usual TEG, Due to the higher temperature difference (up to $400 \mathrm{~K}$ ) compared with $50 \mathrm{~K}$ by usual TEG, there are no available test systems on the market [4][6]. The aim of the evaluating system, described in this paper, is to produce the constant temperature difference, controlled by the host PC in order to measure the TEG electrical parameters built with new materials that support higher temperature differences.

\section{Design and Construction of the HTCM Applied to Micro TEGs}

To investigate and analyze high temperature micro TEGs it is necessary to measure the efficiency of the thermoelectric materials at different temperatures. This section describes the design of the HTCM (high temperature control monitoring) applied to micro TEG parameter measurement.

The developed HTCM has to generate a constant temperature value from $300 \mathrm{~K}$ to $700 \mathrm{~K}$ on the hot side of the TEG during the measurement of the TEG parameters. Temperature settings should be transmitted by an external control device (usually the host PC). Current temperature values are transmitted back from HTCM to control device in order to use the right moment for TEG parameter measurement. Such communication between HTCM and control device make possible the automatically measurement of the TEG parameter through the full temperature range.

The HCTM embedded system was designed and implemented in order to test new TEG materials by specified high temperature ranges and to acquire and transmit data to a host PC. The temperature regulation, especially on high temperatures, means the high electrical power operating, what can't be controlled directly by the host PC. The developed HTCM contains power semiconductor switches with proper driver and protection circuits. These circuits are controlled by embedded microcontroller, which allow the real-time operation of the HTCM device. The host PC would have enough processing power for this task, but usual operating systems don't have the real-time capability, which is very important for safety working of the power circuit. The task sharing between the control device and the HTCM allows the stable operation even by high electromagnetic interference.

The following requirements for the developed HTCM were worked out:

1) Temperature control accuracy: $3 \mathrm{~K}$;

2) Temperature range: $300 \mathrm{~K}$ to $700 \mathrm{~K}$;

3) Heating element power: $500 \mathrm{~W}$;

4) USB interface for the data exchange between $P C$ and microcontroller and firmware update.

By increasing the power, heating process will be going rapidly, and the time to achieve the new temperature setpoint will decrease. The new value of the output power, different from the default value, will cause the recalculating of the PI-controller parameters. The HCTM was developed basing on microcontroller with USB interface to ensure easy firmware upgrade via bootloader system. In addition there is some free software for this system including editor, compiler and programming environment and also bootloader for easy firmware upgrade without external programming device. Figure 2 shows the functional diagram of the proposed HTCM.

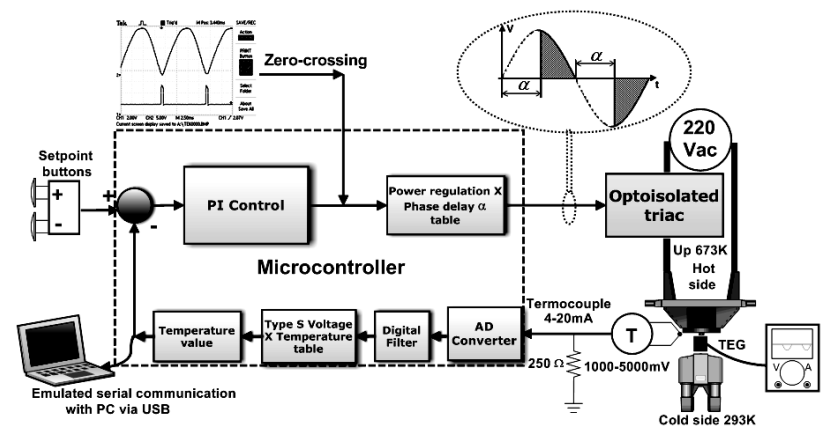

Fig. 2. Functional diagram of the proposed HTCM.

The built-in USB-interface of the microcontroller is used for the connection to the $\mathrm{PC}$ and updating the microcontroller firmware. This allows the easy reprogramming of the HTCM without an external programming device and special programming interfaces. In order to realize the micro TEG performance measurement, the HTCM heats the TEG hot side. To keep the cold side at a constant temperature of approximately $293 \mathrm{~K}$ regulated chiller equipment is used. For the exact temperature measurement in the range from $300 \mathrm{~K}$ to $700 \mathrm{~K}$ a type $\mathrm{S}$ thermocouple was used. The measurement amplifier used together with the thermocouple [12] has the built-in cold side compensation circuit and produces an output current signal of 4 to $20 \mathrm{~mA}$ for the whole temperature range of the thermocouple. This output current signal is transformed to DC voltage (1000 to $5000 \mathrm{mV})$ using a $250 \Omega$ precision resistor. Then it is converted in a digital form by the built in 10-bit ADC.

The ADC converted values are processed by a programmed digital 1th order FIR (finite impulse response) filter contained in the firmware microcontroller 
in order to eliminate peak values and electrical noise. Because the output signal of the $\mathrm{S}$ type thermocouple is not linear, the obtained digital codes have to be transformed into temperature values using a coefficient table. These coefficients for the whole thermocouple temperature range $\left(0\right.$ and $\left.1200{ }^{\circ} \mathrm{C}\right)$ are saved in the microcontroller program memory and have to be applied at each temperature measurement in order to get the correct temperature values. Besides the measuring temperature, the HTCM needs also the set-point temperature settings which correspond to the desired temperature on the TEG, achieved by the temperature control process. These temperature settings can be either transmitted from the host PC or an external control device via communication interface, or can be directly entered by the operator from the key pad.

Both, current temperature and temperature settings are shown on the LCD screen on the top side of the HTCM. This screen is also used for controlling the actual state of the HTCM. By changing temperature settings by the control device, actual value of these settings will be shown in real time on the LCD display.

The HTCM output signal is calculated from the difference between the temperature settings and the current temperature. This difference, also called temperature error, is used in the PI-regulator to calculate the output signal which is to be converted in the output power.

Power switch (optoisolated triac) is used to connect the line voltage to the heating element. The temperature control is taken by switching the line voltage in calculated time moments on each AC voltage period. Because the heating element is powered by $\mathrm{AC}$ voltage, the switching off of the line voltage is done automatically by crossing the zero value. The desired energy amount for the heating element is controlled by manipulating of the phase delay between voltage switching moments.

These moments of the zero crossing value by the line should be established by the external circuit called zerocross detector circuit. The AC voltage from the transformer connected to the bridge rectifier allows to detect each half wave. The rectified voltage gets to the resistance divider and through to low-pass filter connected to the basis of the bipolar transistor. The output signal from the collector of the bipolar transistor is connected to the microcontroller interrupt pin; it is used to calculate the right moment for turning on the triac. In Figure 3 are shown the developed HTCM board with interface connectors, a power system and power control components. The board is developed using also SMD components that is why some discrete components like resistor or capacitor are placed on the bottom side.

According to Figure 3, the developed HTCM board is composed of a power triac (1), an optocoupler triac driver (2), a RS-232 interface (3), an USB interface (4), an output for optional cooler connection (5), S-type thermocouple interface (6), liquid crystal display interface (7), microcontroller (8), keyboard interface (9), programming mode push-button (10) and zero-cross input circuit (11).

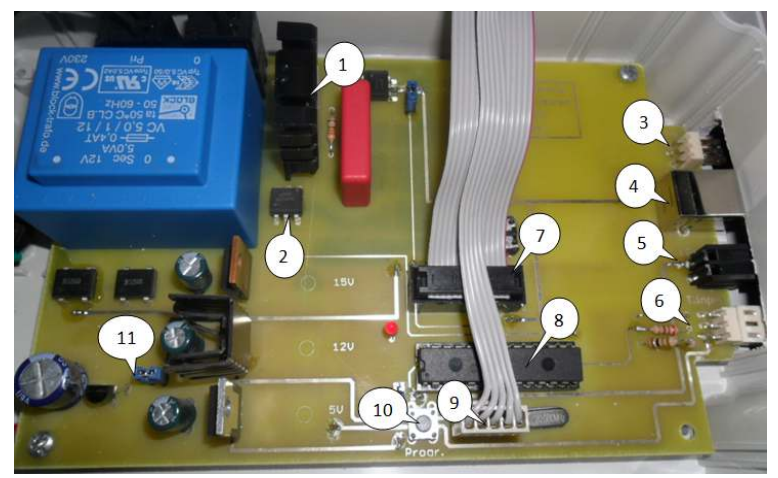

Fig. 3. Developed HTCM board.

The complete system consists in a HTCM (1), micro TEG (2) with hot (3) and cold (4) side and thermocouple temperature sensor (5) is shown on Figure 4.

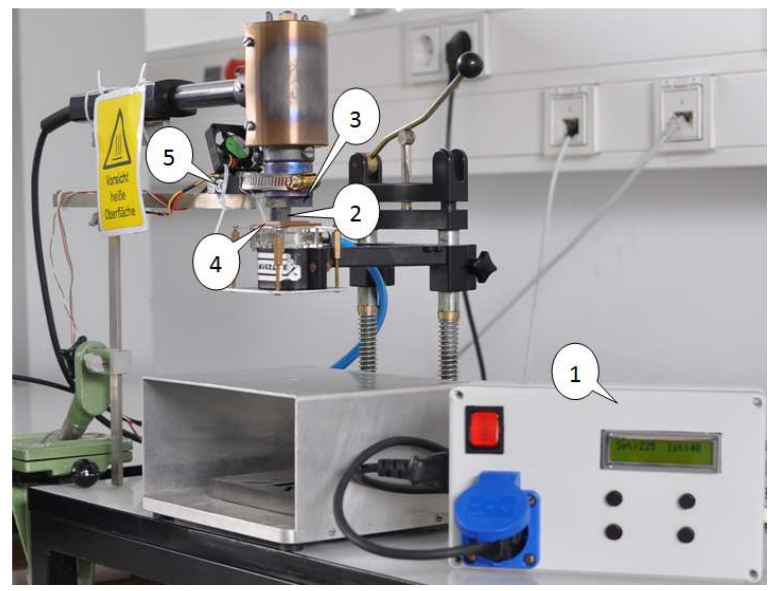

Fig. 4. HTCD with heating device and micro TEG.

\section{Programming and Debugging Tools}

The data acquisition and monitoring systems are applied in various stages of microgeneration such as, for example, in predicting generation failures, practical verification of design data and optimization of the generation efficiency, given that it is possible to inform in real time, the generation parameters and operation.

In this sense, this paper describes an online monitoring system, programmed on free software and data acquisition systems built with electronic components. These electronic sensing microcontrolled systems were built and programmed with the ability to update the firmware wirelessly via bluetooth or zigbee protocols. Based on the discussed system, was used a embedded system based on the microcontroller with USB interface and software for the development of embedded systems, i.e., systems that manipulate data inside another larger system, as is the case of monitoring systems [13]-[14] and of the proposed HTCM.

The programming tool used in this project is called SanUSB and can update the PIC microcontroller firmware via USB channel and is executable in Linux, 
Mac OSX and Windows ${ }^{\circledR}$ operating systems. It is available from the archives at http://www.tinyurl.com/SanUSB [15]. The SanUSB tool enables user-friendly programming and also debugging directly through the open source [16] virtual serial emulation. This can be created quickly and effectively at the very moment the microcontroller is connected directly to a PC, through the USB interface. Equipments designed and built for specific applications tend to be less costly, enhance performance and provide user-friendly environment for control and communication [17]-[19].

\section{Mathematical Model of the HTCM}

Commercially available PI- or PID-controller use the strategy to switch the output on and off only once in second or even rarely. This procedure may be a good method for soldering stations or some similar devices, but it may cause output temperature pulsing in measurement embedded systems like the HTCM. For this reason the HTCM uses another strategy, dosing the output power in each half-wave AC period.

As the heating device supply voltage has a sinusoidal waveform, the linear phase sweep of the supply voltage will have as a result nonlinear power sweep on to the heating element. The developed mathematical model is used to calculate the desired values of the linear phase angle as a function of a linear output power in order to compensate the nonlinearity of the supply voltage. The HTCM embedded system controller works stable as designed with the calculated values of the phase angle available in a microcontroller firmware table.

By triac phase control, used for temperature regulation the triac will automatically switch off by the end of each halfwave of the supply voltage. The triac switching on is done by a command from the microcontroller according to the calculated phase delay. The triac switching on cannot happen for any calculated moment, because the microcontroller is digital device that clocked by defined frequency and can operate only within discrete time moments. Time interval for phase delay is generated by a timer interrupt counter (microcontroller periphery device) and can operate with only some hundreds discrete interrupts in each sinusoidal half-wave period $(10 \mathrm{~ms})$. Larger interrupt frequency will be limited by the processing capacity of the microcontroller CPU, which is also used for other real-time tasks, like temperature measurement and periphery interface controlling. Through practical experiments was established, that the interrupt frequency of 500 times in each sinusoidal half-wave period, does not noticeably affect the execution of other real-time tasks in the developed embedded system.

By varying the number of the interrupts counter (and also the phase angle $\alpha$ ), the output power delivered to the heater $\left(\mathrm{P}_{\mathrm{del}}\right)$ will also change [20]. If there is no delay applied $(\alpha$ $=0)$, the triac will be switching on immediately after zerocrossing detection, the power delivered to the heater will be maximal, calculated by equation (1):

$$
\text { Pdel }=\frac{V_{r m s}^{2}}{R}
$$

where $\mathrm{V}_{\mathrm{rms}}$ is the real value of the supply voltage and $\mathrm{R}$ is the ohmic resistance of the heating device. The simplest method to control the output power is the linear change of the phase angle used for switching on the triac. In this case, the digitalized model of the phase angle change as a function of the timer interrupt counter $(\mathrm{x})$ by the maximal counter value $\mathrm{N}$ (pro half-cycle), is expressed by equation (2):

$$
\text { real }_{\text {phase }}(x)=\frac{\pi \cdot x}{N}
$$

So, the output power that can be expressed by equation (3), described as real_power:

$$
\text { real }_{\text {power }}(x)=\int_{\text {real }_{\text {phase }}(x)}^{\pi} \sin (x) d x
$$

The integral in this expression represents the total power from the triac open moment until the end of the halfcycle. This expression is described as real power, because the dependence of the output power on the variable $\mathrm{x}$ is not linear in this case. The ideal power will be described by the linear changing (decreasing) of maximal output power as function of variable $\mathrm{x}$, as can be seen from equation (4).

$$
\text { ideal }_{\text {power }}(x)=\int_{0}^{\pi} \sin (x) d x-\frac{\int_{0}^{\pi} \sin (x) d x \cdot x}{N}
$$

where $\int_{0}^{\pi} \sin (x) d x$ is the maximal output power for each half-cycle.

Through practical experiments with a PI control response it has been shown that the division of the output power in 300 equal parts is enough for achieving the desired accuracy of temperature regulation. It means that the value of $\mathrm{N}$ is 300 and the value $\mathrm{x}$ can be changed from 0 to $\mathrm{N}$. The linear regulation of the output power is not possible by linear regulation of the phase angle delay. A special dependence needs to be deduced to calculate the phase delay values according to the desired part of the output power. Graphs of the real and ideal power model are shown in Figure 5. The maximum power value is 2 because the integral of $\sin (\mathrm{x})$ over the half period equals 2.

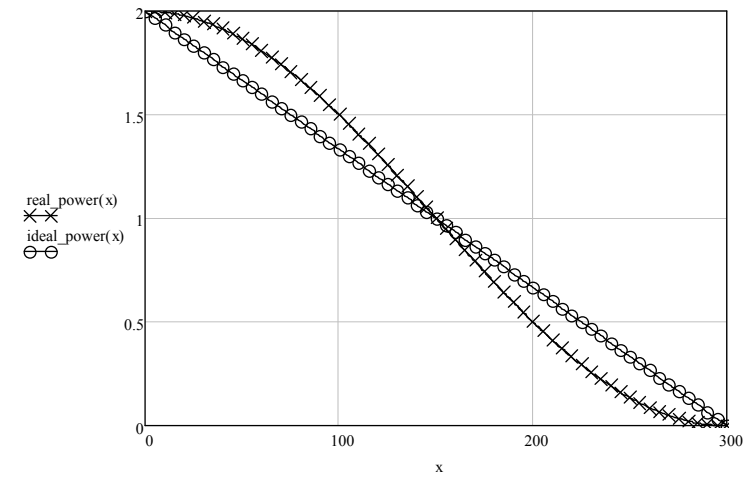

Fig. 5. Graphs of real and ideal power model.

The relative error between the ideal und real power model (in percent) expressed in equation (5) is shown in Figure 6. It is noted that the maximum error introduced between the linear power changing model and linear phase changing model is about $10 \%$. 


$$
\text { power }_{\text {error }}(x)=\frac{\left(\text { real }_{\text {power }}(x)-\text { ideal }_{\text {power }}(x)\right) \cdot 100}{\int_{0}^{\pi} \sin (x) d x}
$$

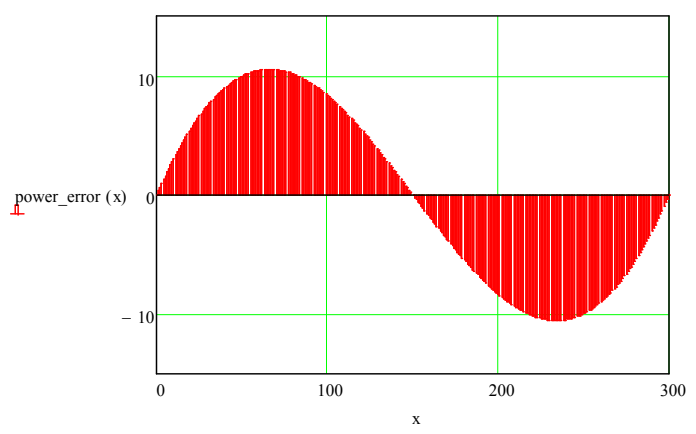

Fig. 6. The relative error between ideal und real power model (in percent).

To calculate the exact phase delay for switching on the triac, the function called ideal phase is presented in equations (6) and (7), obtained from the ideal power equation through a function analogous to equation (3).

$$
\begin{array}{r}
\text { ideal }_{\text {phase }}(x)=a \cos \left(\text { ideal }_{\text {power }}(x)-1\right) \\
\text { ideal }_{\text {phase }}(x)=a \cos \left(\int_{0}^{\pi} \sin (x) d x-\frac{\int_{0}^{\pi} \sin (x) d x \cdot x}{300}-1\right)
\end{array}
$$

In this ideal case there is no linearization error and the power division is optimal. But the triac can't be switched by the microcontroller for any calculated time moment. The microcontroller operates only with some predefined discrete time moments, and the calculated phase value by the equation (7) has to be rounded to the next discrete time moment. It is obvious that such rounding procedure will cause an error in the output power value. It is also obvious that more than $\mathrm{N}$ discrete time moments are needed to represent $\mathrm{N}$ rounded values of output power. The case, when $\mathrm{N}$ is equal to 300 values of output power, are discussed and represented by phase delay, rounded to $\mathrm{T}$ equal to 500 possible values of phase delay. The 500 possible values of phase delay correspond to timer interrupts occurred each $20 \mu \mathrm{s}(10 \mathrm{~ms} / 500)$ after the zero crossing identification. Then, there are just 500 interrupts per each sine half wave, to represent 300 values on output power. Thus the rounded to the next timer interrupts phase by the maximal interrupt value $\mathrm{T}$ per each half wave expressed in equation (8).

$$
\operatorname{dig}_{\text {phase }}(x)=\operatorname{round}\left(\frac{\text { ideal }_{\text {phase }}(x) \cdot T}{\pi}\right)
$$

The function for the resultant output power using the rounded values of phase delay is shown in expression (9). The calculated values of this function are presented as a table and saved in the microcontroller program memory. This table is used by calculating the corrected value of output power.

$$
\operatorname{dig}_{\text {power }}(x)=\int_{\frac{d i g_{\text {powax }}(x) \cdot \pi}{T}}^{\pi} \sin (x) d x
$$

The resultant error between ideal und rounded linear power model (in percent) is expressed in equation (10) and shown in Figure 7. It is noted that the maximum theoretical error between the ideal and rounded power model is less than $0.2 \%$.

$$
\text { power }_{\text {error }}(x)=\frac{\left(\operatorname{dig}_{\text {power }}(x)-\text { ideal }_{\text {power }}(x)\right) \cdot 100}{\int_{0}^{\pi} \sin (x) d x}
$$

The model values of the digitalized rounded linear power model are transformed in integer values and inserted in the microcontroller firmware as a table. It is noted that the maximum error between the ideal and rounded power model is less than $0.2 \%$. Through the discussed mathematical model is possible to reduce the linearization error of $10 \%$ to less than $0.2 \%$.

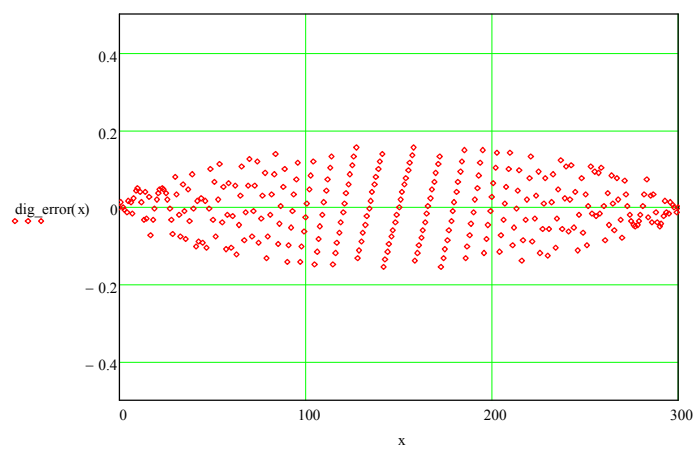

Fig. 7. The resultant error between ideal und rounded linear power model (in percent).

\section{Application of the HTCM with a High Temperature micro TEG}

The graph in Figure 8 shows the implementation of the developed HTCM for measuring the parameter of hightemperature TEG. In this case was used the controlled temperature difference up to $400 \mathrm{~K}$. The graph shows typical values of the internal resistance $(\Omega)$, open-circuit voltage $(\mathrm{mV})$, short-circuit current $(\mathrm{mA})$, TEG output power $(\mu \mathrm{W})$ and temperature difference parameter $(\mathrm{K})$ on the TEG.

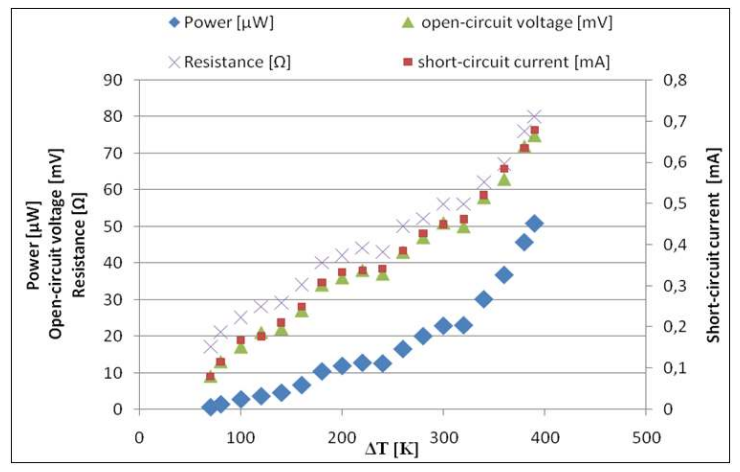

Fig. 8. Values of the internal resistance $(\Omega)$, open-circuit voltage $(\mathrm{mV})$, short-circuit current $(\mathrm{mA})$, TEG output power $(\mu \mathrm{W})$ and temperature difference parameter $(\mathrm{K})$ on the TEG. 


\section{Conclusions}

The developed HTCM operates with a PI temperature regulation realized by a microcontroller system with optoisolated triac output. The temperature can be set by the keypad, or can be received from an external control device by the communication interface. Such communication between HTCM and control device allows fully automatic TEG parameter measurement through the complete temperature range.

For the measurement of the TEG performance, the temperature difference was obtained with the HTCM that holds the TEG hot side at a constant temperature, within temperature of the cold side of approximately $293 \mathrm{~K}$. To realize the exact balancing of the output power, a special mathematical model for the control of the output power was elaborated. Based on changing the triac switching on moments, this model takes into account nonlinearity of $\mathrm{AC}$ supply voltage. As a base point for phase shifting the signal from zero-crossing detector circuit is used, connected to the microcontroller input pin.

The developed mathematical model of the phase change allows the exact output power balancing versus linear phase changing of the supply voltage. Finally, the implemented HTCM works also online with a good performance, reliability and easy firmware updating.

\section{References}

[1] P.H. Kao, P.J. Shih, C.L. Dai, M.C. Liu, "Fabrication and Characterization of CMOS-MEMS Thermoelectric Micro Generators". Sensors 2010, 10, pp. 1315-1325.

[2] R. Ahiska, S. Dislitas, "Microcontroller Based Thermoelectric Generator Application", G.U.J. of Sci. 2006, 19, pp. 131-146.

[3] G.J. Snyder, Small Thermoelectric Generators. The Electrochemical Society Interface 2008, pp. 54-56.

[4] E. Schwyter, W. Glatz, L. Durrer, C, Hierold. "Flexible micro thermoelectric generator based on electroplated Bi2+xTe3-x", in Proc. DTIP of MEMS \& MOEMS, Zurich, SWI (2008), pp. 9-11.

[5] H.S. Han, Y.H. Kim, S.Y. Kim, S. Um, J.M. Hyun, "Performance Measurement and Analysis of a Thermoelectric Power Generator", in Proc. IEEE Thermal and Thermomechanical Phenomena in Electronic Systems, Las Vegas, NV, USA (2010), pp. 1-7.

[6] E. Schwyter, W. Glatz, L. Durrer, C. Hierold, "Bi2Te3Based flexible micro thermoelectric generator with optimized design”, J. Microelectromech, Sys, 2009, 18, pp. 763-772.

[7] A. Kucukkomurler, "Thermoelectric Power high temperature wireless sensing", J. Thermal Sci. and Techn. 2009,4 , pp. 63-73.

[8] B. Jang, S. Seungwoo Han, J. Kim, "Optimal design for micro-thermoelectric generators using finite element analysis", Microelectronic Engineering 2011, 88, 775-778.

[9] R.Y. Nuwayhid, D.M. Rowe, G. Min, "Low cost stove-top thermoelectric generator for regions with unreliable electricity supply", Renew. Energy 2003, 28, pp. 205-222.

[10] Multi-Mission Radioisotope Thermoelectric Generator Heat Exchangers for the Mars Science Laboratory Rover, Available

online: http://ntrs.nasa.gov/archive/nasa/casi.ntrs.nasa.gov/201200 06581 2012006676.pdf (accessed on 03 Jan 2013).
[11] Thermoelectric Power Generation Using Waste-Heat Energy as an Alternative Green Technology, Available online:

http://www.benthamscience.com/eeng/samples/eeng21/0004EENG.pdf (accessed on 03 Jan 2013).

[12] Rössel Messtechnik, "Analog transmitters in 2-wire technique", Available online: http://www.roesselmesstechnik.de/images/downloadsection/manuals/english /KAT_BED_eng.pdf (accessed on 03 Dez 2012).

[13] S.C.S. Juca, P.C.M. Carvalho, F.T. Brito, "A Low Cost Concept for Data Acquisition Systems Applied to Decentralized Renewable Energy Plants". Sensors 2011, 11 , pp. 743-756.

[14] F.T. Brito, S.C.S. Juca, P.C.M. Carvalho, "Contrologger: A remote monitoring system for descentralized renewable energy sources". Renewable energy \& Power Quality Journal 2012, 10, p.432.

[15] SanUSB Tool, Available online: http://www.tinyurl.com/SanUSB (accessed on 02 Oct 2012).

[16] J.W. Paulson, G. Succi, A. Eberlein, "An empirical study of open-source and closed-source software products", IEEE Trans. Software Eng. 2004, 30, pp. 246-256.

[17] Belmili, H.; Cheikh, S.; Haddadi, M.; Larbes, C. "Design and development of a data acquisition system for photovoltaic modules characterization". Renewable Energy 2010, 35, pp. 1484-1492.

[18] M. Balan, M. Damian, L. Jäntschi, "Preliminary results on design and implementation of a solar radiation monitoring system". Sensors 2008, 8, pp. 963-978.

[19] S. Rosiek, F.J. Batlles, "A microcontroller-based dataacquisition system for meteorological station monitoring Original Research Article Energy Conversion and Management", Volume 49, Issue 12, December 2008, pp. 3746-3754

[20] D. Ibrahim, Microcontroller based temperature monitoring and control, Elsevier Science \& Technology Books, 2002. 